\title{
Formation of the Knowledge Base on the basis of Modeling the Technological Process with the Use of Fuzzy Logic

\author{
${ }^{1}$ Larisa A. Simonova, ${ }^{2}$ Ildar R. Davletshin \\ 1,2 Kazan Federal University \\ Email: lasimonova@mail.ru, ildar_davletshin@mail.ru
}

Received: 20 ${ }^{\text {th }}$ August 2019, Accepted: $30^{\text {th }}$ September 2019, Published: $31^{\text {st }}$ October 2019

\begin{abstract}
This article is devoted to the formation of a knowledge base of an intellectual add-on for technological process control systems based on modeling using fuzzy logic and a use-case system. The intelligent add-on is a multi-agent system that includes the agents of a different nature. This article focuses on agents working on fuzzy logic. The knowledge base is part of an intelligent control system and, in relation to production conditions, consists of a set of production rules that describe the dependence of the main indicators of the technological process. The study identified the main parameters of the melting process, as one of the processes that determine the quality of the finished product. A review of sources describing the production technology is carried out and rules are highlighted. Linguistic variables are defined and ranges of values in which they change are defined. The stages of obtaining a solution using fuzzy logic are described. A mathematical model has been created that is suitable for choosing the optimal process conditions and for integration into an intelligent superstructure as one of the components. The structure of the precedent is described, the algorithm is briefly described in the steps of selecting precedents, adding new precedents to the database.
\end{abstract}

\section{Keywords}

Fuzzy Logic, Artificial Intelligence, Intelligent Tuning, Automation, Rules, Use Cases.

\section{Introduction}

In modern conditions, the leading manufacturers of heat-insulating materials use automatic production lines, on which control tasks are carried out mainly with the help of SCADA-systems, however, there are still tasks that must be solved by a person.

One of the further ways of developing automated production systems is the use of artificial intelligence and the creation of an intelligent add-on based on a multi-agent system [1] consisting of a coordinator agent and agents working on fuzzy logic [2] or neural networks. The advantage of multi-component systems is the absence of restrictions imposed on the nature of an agent. It can be a software module, electronic or physical mechanism. This feature allows you to combine different methods of emulating artificial intelligence when creating an intelligent add-on for a control system.

Such an add-on allows improving existing management systems to address management issues in emergency situations.

\section{Methods}

An intelligent add-in requires the creation of a rule base and a use case database. The rule base is a set of rules that describe the dependence of production parameters under various conditions. To create such a base in this paper, it is proposed to use fuzzy logic that allows formulations friendly to ordinary human perception [3]. The use case database contains reference sets of process parameters for each operation.

\section{Results and Discussion}

Building a knowledge base. When analyzing the technological process for the production of heat-insulating materials, for example, the melting process was selected, This process is the first step and largely determines the quality of the finished product. It consists of the following operations: dosing and preparation of raw materials; melting.

The key work parameters related to this process are: fraction and strength of raw materials and fuels, chemical composition of the material, ratio of raw materials/fuels, smelter capacity, blast air supply, product acidity module, melt viscosity, melt temperature, melt surface tension; the speed and the ratio of the revolutions of the rolls of the fiber formation unit and the cooling intensity (for the centrifugal-blast method for producing fiber) [4].

Most parameters are interconnected. The following dependencies exist:

- "The greater the volume of blown air, the lower the fuel consumption";

- "The greater the deviation of the fraction of raw materials from the optimal interval, the lower the productivity of the smelter";

- "The more silicon and aluminum oxides in the feed, the higher the melt viscosity [4]."

- The higher the pressure in the tuyere of the cupola, the lower the temperature of the melt;

- The higher the height of the charge column, the higher the temperature of the melt and lower the temperature of the exhaust gases [5]. 
When creating rules, the logical operators "AND", "OR", "IF" and linguistic variables are used: "many", "small", "strong", "weak", "medium", "high", "low" [6 ]. For all linguistic variables (another term name), numerical intervals are specified. The rule consists of antecedent (condition) and consequent (action) [7].

Working with fuzzy logic consists of the following steps:

Phasification - determining the degree to which specific values of production parameters read from sensors or specified by the operator belongs to certain linguistic variables of the condition (antecedent).

A fuzzy conclusion is the determination of the degree to which the result belongs to certain linguistic variables of the action (consequent).

Defazification - the transition from fuzzy values to specific parameters that are fed to actuators or issued as recommendations to operators.

All rules are shared between agents of a multi-agent system. For clarity, the agents are summarized in the diagram in Figure 01 [4].

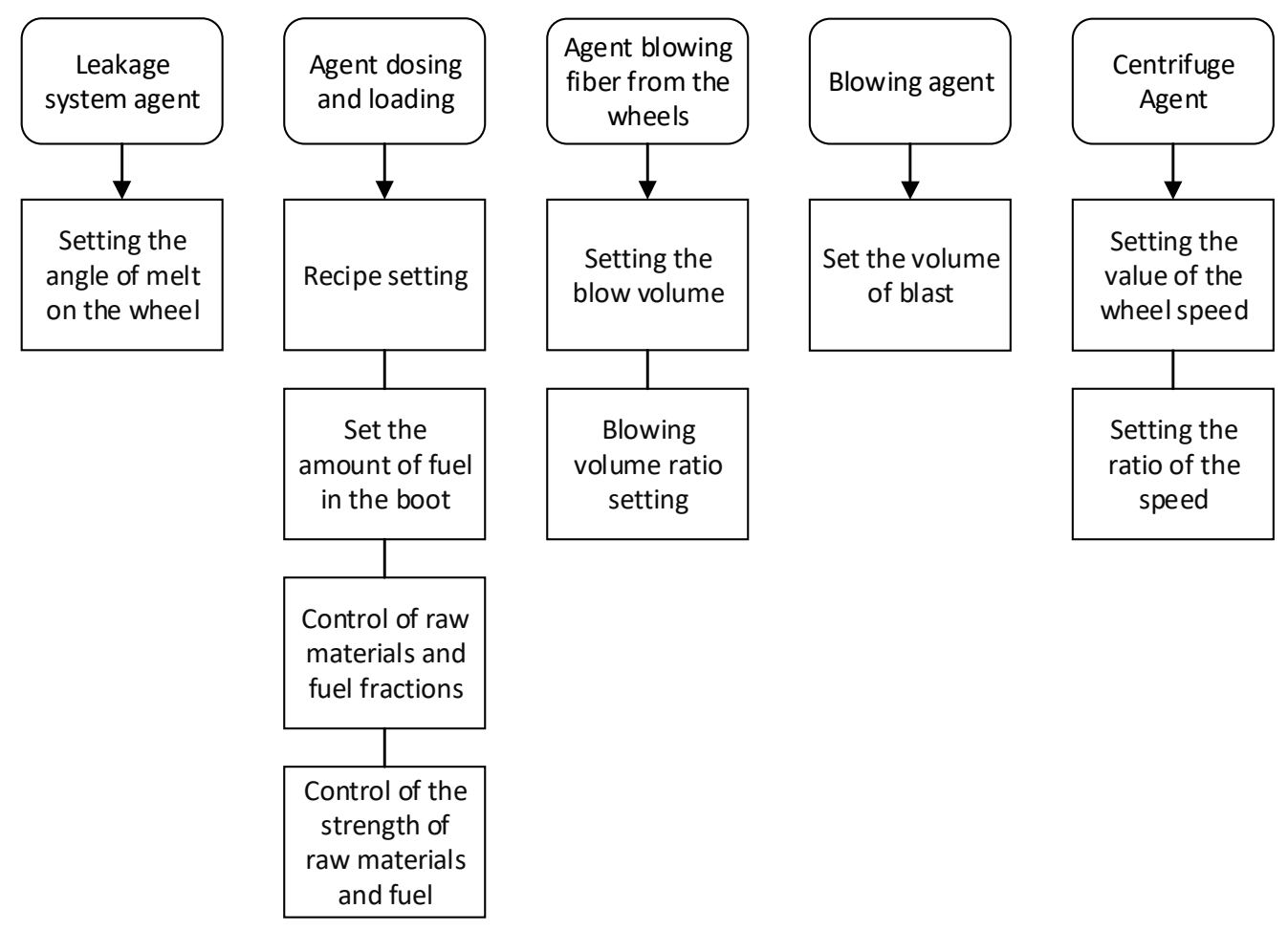

Figure 1: Agents Tasks

We carry out the phasing of the parameters of the control object. To calculate the membership function, we use the triangular membership function:

$$
\mu_{A}(x, a, b, c)=\left\{\begin{array}{c}
0, x \leq a, \\
\frac{x-a}{b-a}, a \leq x \leq b, \\
\frac{c-x}{c-b}, b \leq x \leq c, \\
0, c \leq x .
\end{array},\right.
$$

where a, c is the measuring range of the variable;

$\mathrm{x}$ is the variable corresponding to the output linguistic variable and belonging to the range from a to $\mathrm{c}$;

$\mathrm{b}$ is the most probable value of the variable.

Defazification by the method of the center of gravity is performed according to formula 2.

where $\mathrm{y}$ is a clear conclusion or result of defazification

$$
y=\frac{\int_{x \min }^{x \max } x \mu(x) d x}{\int_{x \min }^{x \max } \mu(x) d x},
$$

$\mu(x)$ - membership function of a fuzzy set.

We form the ranges of input and output parameters [4]: 


\begin{tabular}{|c|c|c|c|}
\hline Linguistic variable & Low & - optimum & High \\
\hline Range & $<1350$ & {$[1350-1400]$} & $>1400$ \\
\hline
\end{tabular}

Table 1: Melt Temperature. Ranges of Change 1300-1400 gr. C (valid for one type of charge).

$$
\begin{gathered}
\mu_{A}(x, a, b, c)=\left\{\begin{array}{c}
0, x \leq 1200 \\
\frac{x-1200}{1375-1200}, 1200 \leq x \leq 1375, \\
\frac{1550-x}{1550-1375}, 1375 \leq x \leq 1550 \\
0, c \leq x
\end{array}\right. \\
\mu_{A}(x, a, b, c)=\left\{\begin{array}{c}
0, x \\
\frac{x-8}{13-8}, 8 \leq x \leq 13 \\
\frac{18-x}{18-13}, 13 \leq x \leq 18 \\
0,18 \leq x
\end{array}\right.
\end{gathered}
$$

\begin{tabular}{|c|c|c|c|}
\hline Linguistic variable & Low & - optimum & High \\
\hline Range & {$[1200-2000]$} & {$[2000-2800]$} & $(2800-3600]$ \\
\hline
\end{tabular}

Table 2: Melting Performance. Range 1200 - $3600 \mathrm{~kg} /(\mathrm{m} 2 * \mathrm{~h})$

$$
\mu_{A}(x, a, b, c)=\left\{\begin{array}{c}
0, x \leq 1200 \\
\frac{x-1200}{2400-1200}, 1200 \leq x \leq 2400 \\
\frac{3600-x}{3600-2400}, 2400 \leq x \leq 3600 \\
0.3600 \leq x
\end{array}\right.
$$

\begin{tabular}{|c|c|c|c|}
\hline Linguistic variable & Low & - optimum & High \\
\hline Range & {$[45-50]$} & {$[50-55]$} & {$[55-60]$} \\
\hline
\end{tabular}

Table 3: The Amount of Blast in the Oven. Range 45-60m3 / (m2 * min)

$$
\mu_{A}(x, a, b, c)=\left\{\begin{array}{c}
0, x \leq 45 \\
\frac{x-45}{52,5-45}, 45 \leq x \leq 52,5 \\
\frac{60-x}{60-52,5}, b \leq x \leq 60 \\
0.60 \leq x
\end{array}\right.
$$

\begin{tabular}{|c|c|c|c|}
\hline Linguistic variable & Low & - optimum & High \\
\hline Range & {$[17-18]$} & {$[18-19]$} & {$[19-20]$} \\
\hline
\end{tabular}

Table 4: The Amount of Fuel in the Load. Range 17-20\%

$$
\mu_{A}(x, a, b, c)=\left\{\begin{array}{c}
0, x \leq 17 \\
\frac{x-17}{18,5-17}, 17 \leq x \leq 20 \\
\frac{20-x}{20-18,5}, 18,5 \leq x \leq 20 \\
0,20 \leq x
\end{array},\right.
$$

\begin{tabular}{|c|c|c|c|}
\hline Linguistic variable & Shallow & Secondary & Large \\
\hline Range & {$[0-20]$} & {$[20-40]$} & {$[40-100]$} \\
\hline
\end{tabular}

Table 5: Fraction of Raw Materials. Range 0-100 mm

$$
\mu_{A}(x, a, b, c)=\left\{\begin{array}{c}
0, x \leq 0 \\
\frac{\mathrm{x}-0}{30-0}, 0 \leq \mathrm{x} \leq 30 \\
\frac{100-\mathrm{x}}{100-30}, 30 \leq \mathrm{x} \leq 100 \\
0,100 \leq x
\end{array}\right.
$$

The rules of the agents are given in table 6 . 


\begin{tabular}{|l|l|}
\hline \multicolumn{1}{|c|}{ Agent } & \multicolumn{1}{c|}{ Rule } \\
\hline $\begin{array}{l}\text { Blast Supply } \\
\text { Agent }\end{array}$ & $\begin{array}{l}\text { If the volume of blast supplied is "low", then the melting capacity is "low"; } \\
\text { If the volume of blast supplied is "average", then the melting capacity is "low"; } \\
\text { If the volume of blast supplied is "high", then the productivity is "high". }\end{array}$ \\
\hline $\begin{array}{l}\text { Dosing and } \\
\text { Loading Agent }\end{array}$ & $\begin{array}{l}\text { If the fuel content is "high", then the productivity is "low", the melt temperature is "high"; } \\
\text { If the fuel content is "average", then the productivity is "average" and the melt temperature is } \\
\text { "average"; } \\
\text { If the fuel content is "high", then the productivity is "low", the melt temperature is "high"; }\end{array}$ \\
\hline
\end{tabular}

\section{Table 6: Agents Rules [4]}

Modeling the work of agents is performed using the Fuzzy logic toolbox [9]. This add-in is specifically designed to work with fuzzy logic. At the first stage, agent parameters are set. The number of inputs and outputs set the type of graph, border.

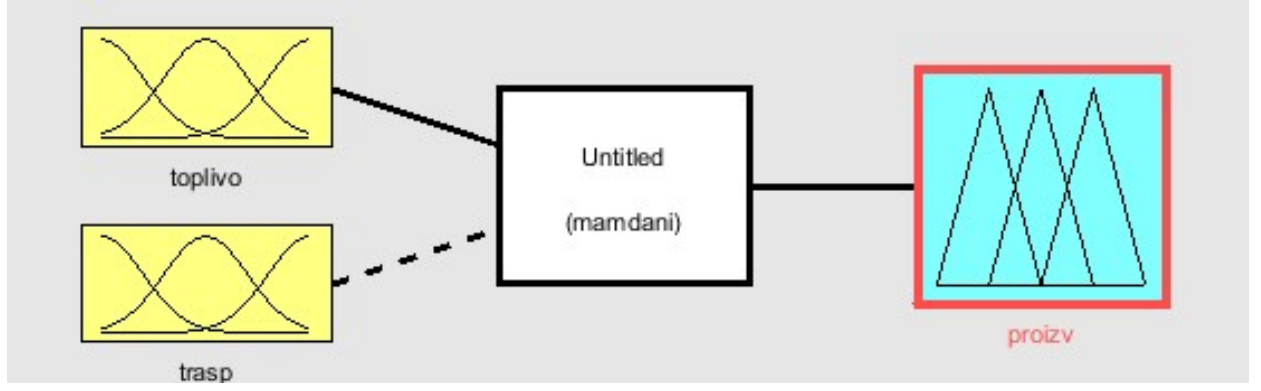

Figure 2 : Fuzzy Logic Toolbox Editor

The following are the rules. The rule editor is shown in Figure 03.

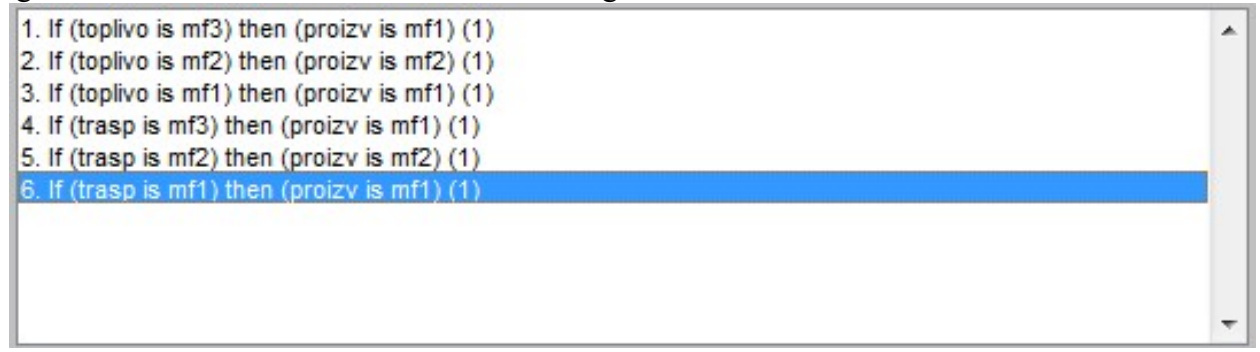

Figure 3: Fuzzy Logic Toolbox Editor

When the rules are created, you can see on the graph how the output parameter will change when one or two input parameters change (when one or more parameters work). A three-dimensional graph is shown in Figure 04.

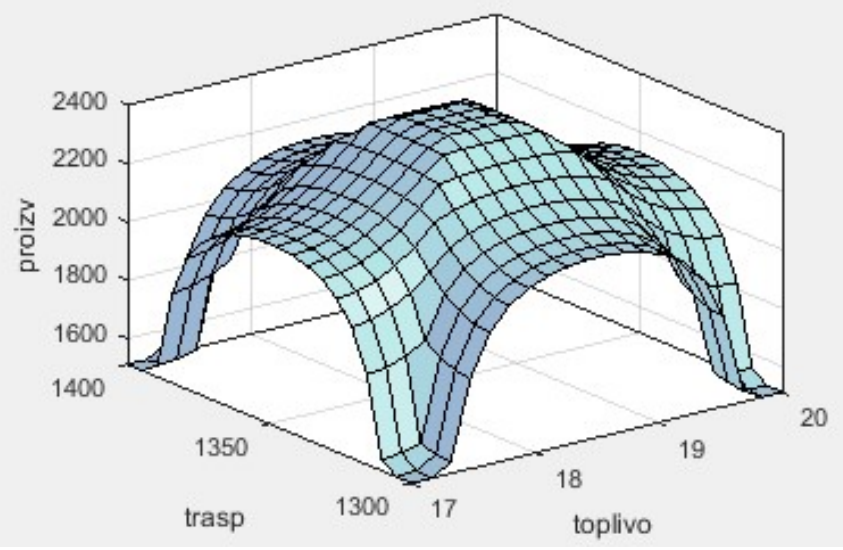

Figure 4: Graphical Representation of the Rules of the Fuzzy Logic Toolbox

The formation of the base of precedents [9]. The base case is an array containing information about the type of product, the parameters of the production process and the weight of the use case. Precedents are the results of previously solved tasks for process control.

Use cases are scanned by the agent coordinator in decreasing order weight. The use case weight is updated after each successful use of the use case. A used precedent increases its weight, non-used ones lower. 
The use case base operator is the agent coordinator. When a situation appears that is not previously described in the use case database when a new use case appears, the agent coordinator includes it in the database. The control problem solvers are agents operating on fuzzy logic and neural networks, the values calculated by them, as well as the response of the system to them are recorded by the agent coordinator and constitute a precedent structure.

An exemplary case-base structure is shown in table 7.

\begin{tabular}{|c|c|c|c|c|}
\hline Use case code & Production & $\begin{array}{l}\text { Reference parameters at the } \\
\text { input of operations }(\mathrm{N})\end{array}$ & $\begin{array}{c}\text { Reference parameters at the } \\
\text { output of operations }(\mathrm{K})\end{array}$ & Use case weight \\
\hline 001_PR1 & PR1 & $\mathrm{N} 1, \mathrm{~N} 2, \ldots, \mathrm{N} \mathrm{n}-1, \mathrm{Nn}$ & $\mathrm{K} 1, \mathrm{~K} 2, \ldots, \mathrm{K}$ n-1, Kn & \\
\hline 002 PR1 & PR1 & $\mathrm{N} 1, \mathrm{~N} 2, \ldots, \mathrm{N}$ n-1, Nn & $\mathrm{K} 1, \mathrm{~K} 2, \ldots, \mathrm{K}$ n-1, Kn & \\
\hline 001_PR2 & PR2 & $\mathrm{N} 1, \mathrm{~N} 2, \ldots, \mathrm{N}$ n-1, Nn & $\mathrm{K} 1, \mathrm{~K} 2, \ldots, \mathrm{K}$ n-1, Kn & \\
\hline 001_PR3 & PR3 & $\mathrm{N} 1, \mathrm{~N} 2, \ldots, \mathrm{N}$ n-1, Nn & $\mathrm{K} 1, \mathrm{~K} 2, \ldots, \mathrm{K}$ n-1, Kn & \\
\hline & $\ldots$ & $\ldots$ & $\ldots$ & \\
\hline 001_PRn & $\overline{\text { PRN }}$ & $\mathrm{N} 1, \mathrm{~N} 2, \ldots, \mathrm{N}$ n-1, Nn & $\mathrm{K} 1, \mathrm{~K} 2, \ldots, \mathrm{K}$ n-1, Kn & \\
\hline
\end{tabular}

Table 7: Use Case Database Data Structure

Work with the knowledge base is performed according to the following algorithm.

The system receives information about the launch or conversion to a new type of product. The system in accordance with the data of specifications of the issued items available to it forms two sets:

A lot of $P R=(P R 1, P R 2, \ldots, P R n-1, P R n)$, where PR is a specific quality indicator of the product.

The set $K=(K 1, K 2, \ldots, K n-1, K n)$, where $K$ is the reference parameters at the output of each operation of the production process.

Next is an appeal to the use case database, which stores work parameters pre-installed by an expert or added during the training of the system. In addition to the above parameter sets, the precedent contains the third set, the $\operatorname{set} N=(\mathrm{N} 1, \mathrm{~N} 2$, ..., $\mathrm{Nn}-1, \mathrm{Nn}$ ). The $\mathrm{N}$ set is a set of reference parameters, but already at the input of each operation. There can be several precedents for one type of product, each of them is assigned a weight, in accordance with which the order of application of the parameters recorded in them is built. A comparison of the actual values at the output of operations with the reference.

If the sets are equal, the cycle ends. Otherwise, the next precedent in weight is used. If one of the precedents worked, the weights of all others related to this product are recalculated.

If all the settings recorded in the use cases do not allow to optimize the process, then the rules are referred to based on the fuzzy logic described above. A search is made for an agent (rule) that is responsible for a certain operation and can provide a target value of $\mathrm{K}$. If there are several such agents, then several exposure scenarios are generated with their sequential use and control of the reaction of the control object.

If the parameters calculated by the agents also did not optimize and stabilize the process, the third module "ANS Intelligent Agent" is included in the work. This agent continuously, during the entire working time of the system, removes the parameters of the inputs and outputs of operations. It is trained on them and can calculate the necessary control action with the help prepared during the training of the neural network [10].

After the reference parameters at the output of operations are achieved, all parameters are entered into the database, forming a new precedent. The system applies for support to a person (expert or operator) only if none of its blocks has coped with the task.

\section{Summary}

Fuzzy logic allows you to simulate technological processes in the face of uncertainty. This approach, which allows you to use the experience of an expert, allows you to create intelligent process control systems.

\section{Conclusions}

The article lists the main indicators of the melting process, as one of the processes that determine the quality of the finished product. Linguistic variables are defined and ranges of values in which they change are defined. The stages of obtaining a solution using fuzzy logic are described. The use of linguistic variables, fuzzy logic rules, approximate reasoning allows you to bring the expert's experience into the management of technological processes.

\section{Acknowledgments}

The work is performed according to the Russian Government Program of Competitive Growth of Kazan Federal University. 


\section{References}

[1] Dorri, Ali \& Kanhere, Salil \& Jurdak, Raja. (2018). Multi-Agent Systems: A survey. IEEE Access. 1-1 10.1109/ ACCESS.2018.2831228.

[2] Applied fuzzy systems: Trans. from japan./ K. Asai, D. Vataada, S. Ivaiydr .; edited by T. Terano, K. Asai, M. Sugano. - M .: Mir, 1993.368 pages, ill.

[3] Dernoncourt, Franck. (2013). Introduction to fuzzy logic.

[4] A.I. Zhilin. Mineral wool. Edited by V.A. Kitaytsev. State Publishing House of Building Materials Literature Moscow 1953.

[5] K.E. Goryainov, S.K. Gorynova. The technology of heat-insulating materials and products: Textbook for high schools.-M .: Stroyizdat, 1982._376 pages, Il.

[6] R. Rojas: Neural Networks, Springer-Verlag, Berlin, 1996

[7] M.A. Chernova, L.A. Simonova, V.V. Abramova. The use of fuzzy logic in a process control system in a vacuumspraying technological complex // Fundamental Research. - 2014. - No. 12-4. - pp. 744-750.

[8] A.V. Leonenkov. Fuzzy modeling in MATLAB and fuzzyTECH. - SPb .: BHV-Petersburg, 2005.736 pages: ill. 2 .

[9] I.R. Davletshin. L.A. Simonova. V.V. Abramova. Intelligent add-on of the control system of an automatic line for the production of building materials based on a multi-agent system // Scientific and Technical Bulletin of the Volga Region. - Kazan: Scientific and Technical Bulletin of the Volga Region, 2018. №2. - pp. 70-73.

[10] M. Nielsen, Neural Networks and Deep Learning (Determination Press, 2015). 